Research Article

\title{
Joint Transceiver Optimization of MIMO SWIPT Systems Assisted by Reconfigurable Intelligent Surface
}

\author{
Jinxin Zhu $(\mathbb{D}$ and Jun Shao \\ School of Information Technology, Yancheng Institute of Technology, Yancheng 224051, China \\ Correspondence should be addressed to Jinxin Zhu; zjx@ycit.edu.cn
}

Received 10 October 2021; Revised 10 November 2021; Accepted 7 December 2021; Published 25 December 2021

Academic Editor: Fangqing Wen

Copyright ( 2021 Jinxin Zhu and Jun Shao. This is an open access article distributed under the Creative Commons Attribution License, which permits unrestricted use, distribution, and reproduction in any medium, provided the original work is properly cited.

In this work, a reconfigurable intelligent surface (RIS)-assisted multiple-input multiple-output (MIMO) system is studied with wireless energy harvesting (EH). Specifically, we focus on maximizing the harvested power at the receiver by joint optimization of the signal covariance, the phase shifter, and the power splitting factor, subject to the information rate and transmit power constraints. The formulated problem is hard to address due to the nonconcave objective and the nonconvex constraints. To tackle these challenges, an alternating optimization (AO) framework is proposed, where the phase shifter is solved by the penalty-based method. Simulation results validate the performance of the proposed approach.

\section{Introduction}

Recently, the reconfigurable intelligent surface (RIS) has been treated as a promising technique for wireless networks, due to the low cost and energy-efficient merits [1]. Specifically, the authors of $[2,3]$ studied the RIS-assisted multipleinput single-output (MISO) network and multiple-input multiple-output (MIMO) network, respectively, which suggested that joint active beamforming (BF) and phase shifter (passive BF) design can improve the performance of wireless networks. Then, the work in [4] studied the joint active and passive BF design in RIS-aided wiretap wireless channels, which demonstrated the ability of RIS in improving the security of wireless networks.

Besides the effect of improving the spectrum efficiency or expanding the coverage in wireless networks, the application of RIS is also appealing for other application scenarios such as wireless location and radar signal detection. Specifically, the work in [5] studied the near-field passive localization and gain-phase compensation with partly calibrated array. Also, the study in [6] investigated the three-dimensional near-field localization using a spatially spread acoustic vector sensor. Then, the authors of $[7,8]$ studied the joint location and communication design in RIS-aided wireless networks. As for the radar signal detection, in [9], an estimation algorithm for MIMO radar was developed with arbitrary sensor geometry. Also, in [10], the authors proposed a generalized multiple signal classification (MUSIC)-like algorithm for a height measurement with meter wave polarimetric radar. Then, in $[11,12]$, the authors investigated the algorithm design in the RIS-aided radar system, which suggested that RIS can improve the performance of radar target detection. Furthermore, in [13], the authors proposed dual-polarized RIS schemes in MIMO network, which suggested that dualpolarized RIS can further improve the spectrum efficiency in the MIMO network. Besides these works being mainly based on far-field behaviors of RIS, recently in [14], the authors studied the near-field behaviors of RIS and proposed power scaling laws. These works suggest the various application perspectives of RIS.

On the other hand, wireless energy harvesting (EH) has been regarded as an effective approach to improve the energy efficiency (EE) of wireless networks, where EH-enabled wireless devices can harvest energy from either radio frequency (RF) signals radiated by the transmitter (Tx) and recharge their batteries, which is commonly named as simultaneous wireless information and power transfer (SWIPT) $[15,16]$. 
Specifically, the authors of $[15,17]$ studied the SWIPT design with power splitting (PS) and time switching (TS) schemes, respectively. However, the study in [18] investigated the hybrid PS and TS design in the full-duplex MIMO SWIPT channel. Currently, SWIPT has been widely used in various wireless networks such as the wireless sensor network (WSN) [19] and the Internet-of-Things (IoT) network [16].

Moreover, several works investigated the effect of RIS in improving the information and energy transfer in SWIPT work. Specifically, by assuming the individual information receiver (IR) and energy receiver (ER), in [20, 21], the authors investigated the RIS-assisted MISO and MIMO network with SWIPT, where penalty dual decomposition (PDD) and alternating optimization (AO) methods were proposed, respectively. Then, in [22], the authors studied the co-located receiver design in RIS-assisted SWIPT network, where a penalty-based majorization-minimization approach was proposed to optimize the information rate under the $\mathrm{EH}$ constraint. Also, the authors of [23] studied the secrecy SWIPT design with the assistance of RIS, where a penaltybased manifold optimization was proposed.

The aforementioned works about SWIPT network mainly focused on the transmission power minimization or information rate maximization design. In fact, maximizing the harvested power is another kind of objective, which has been less investigated in RIS-assisted SWIPT systems. Motivated by these observations, this work considers a MIMO SWIPT system where a Tx sends information and energy to a receiver (Rx), with the aid of a RIS. To be specific, we study a joint signal covariance, RIS passive $\mathrm{BF}$, and Rx PS ratio design to maximize the harvested power, subject to the information rate constraint and the power budget. The formulated problem is hard to tackle, since the objective is nonconcave and the constraints are nonconvex. To address these challenges, an alternating optimization framework is proposed, where the phase shifter is solved by the penaltybased semidefinite relaxation (SDR) method. Simulation results validate that with proper designed passive BF, RIS can significantly enhance the harvested power at Rx.

Notations. Throughout this work, boldface lowercase and uppercase letters denote vectors and matrices, respectively. The superscripts $T, *$, and $H$ denote the transpose, conjugate, and conjugate transpose, respectively. $\operatorname{Diag}\left(a_{1}, \ldots, a_{N}\right)$ is a diagonal matrix with diagonal elements $a_{1}, \ldots, a_{N}$, while $\operatorname{diag}(\mathbf{A})$ denotes a vector consists of the main diagonal elements of A, respectively. $\operatorname{Tr}(\cdot)$ and $\operatorname{rank}(\cdot)$ represent the trace and rank. Besides, $\mathfrak{R}\{\cdot\}$ denotes the real part of a complex variable. $\mathbf{A} \pm 0$ indicates that $\mathbf{A}$ is positive semidefinite. $\odot$ is the Hadamard product. I is an identity matrix with proper dimension. $\mathscr{C} \mathscr{N}(\mathbf{u}, \Sigma)$ denotes a circularly symmetric complex Gaussian random vector with mean $\mathbf{u}$ and covariance $\Sigma$. $|\cdot|$ denotes the absolute value or the determinant. In addition, $\ln (\cdot)$ denotes the natural logarithm.

\section{System Model and Problem Formulation}

2.1. System Model. We study a RIS-aided MIMO system as illustrated in Figure 1, which have one Tx, one RIS, and one $\mathrm{Rx}$. It is assumed that Tx and $\mathrm{Rx}$ are equipped with $N_{T}$ and

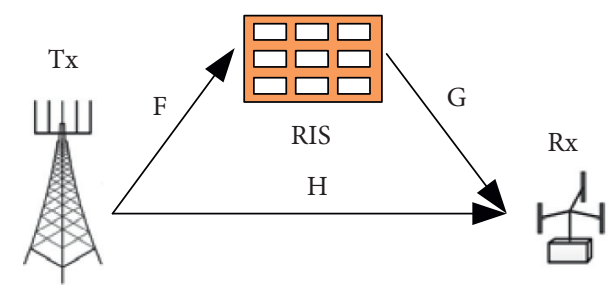

FIgURE 1: RIS-assisted MIMO SWIPT network.

$N_{R}$ antennas, while the RIS has $M$ reflecting elements, respectively. The channel coefficients between $\mathrm{Tx}$ to $\mathrm{Rx}$, Tx to RIS, and RIS to Rx are denoted as $\mathbf{H} \in \mathbb{C}^{N_{R} \times N_{T}}, \mathbf{F} \in \mathbb{C}^{M \times N_{T}}$, and $\mathbf{G} \in \mathbb{C}^{N_{R} \times M}$, respectively.

Let $\mathbf{s} \sim \mathscr{C} \mathscr{N}(\mathbf{0}, \Sigma)$ denotes the transmit signal with $\Sigma \in \mathbb{C}^{N_{T} \times N_{T}}$ being the covariance. Then, the received signal at the $\mathrm{Rx}$ is

$$
\mathbf{y}=(\mathbf{H}+\mathbf{G \Theta F}) \mathbf{s}+\mathbf{n}_{r},
$$

where $\mathbf{n}_{r} \in \mathbb{C}^{N_{R} \times 1}$ is the noise vectors at $\mathrm{Rx}$ with $\mathbf{n}_{r} \sim \mathscr{C} \mathscr{N}_{r}\left(0, \sigma_{r}^{2} \mathbf{I}\right)$.

The phase shifter matrix of the RIS is denoted by $\Theta=\operatorname{Diag}\left(\theta_{1}, \ldots, \theta_{m}\right)$, with $\theta_{m}$ denoting the reflecting coefficient (RC) of the $m$-th element satisfying $\left|\theta_{m}\right|^{2}=1$.

Rx utilizes a PS ratio $\rho$ to divide the received signal into two streams, which is given by

$$
\begin{aligned}
& \mathbf{y}_{I}=\sqrt{\rho}\left((\mathbf{H}+\mathbf{G \Theta F}) \mathbf{s}+\mathbf{n}_{r}\right)+\mathbf{n}_{p}, \\
& \mathbf{y}_{E}=\sqrt{1-\rho}\left((\mathbf{H}+\mathbf{G} \Theta \mathbf{F}) \mathbf{s}+\mathbf{n}_{r}\right),
\end{aligned}
$$

where $\mathbf{n}_{p} \in \mathbb{C}^{N_{R} \times 1}$ is the processing noise at $\mathrm{Rx}$ with $\mathbf{n}_{p} \sim \mathscr{C} \mathscr{N}\left(0, \sigma_{p}^{2} \mathbf{I}\right)$.

Thus, the harvest energy at $\mathrm{Rx}$ is given by

$$
E(\boldsymbol{\Sigma}, \boldsymbol{\Theta}, \rho)=(1-\rho)\left(\operatorname{Tr}\left(\boldsymbol{\Sigma} \hat{H} \stackrel{\wedge}{\mathbf{H}}^{H}\right)+N_{R} \sigma_{r}^{2}\right),
$$

where $\hat{\mathbf{H}}=\mathbf{H}+\mathbf{G \Theta F}$ is the equivalent channel from Tx to $\mathrm{Rx}$, and we assume the unit energy conversion efficiency at the $\mathrm{Rx}$.

Accordingly, the information rate is

$$
R(\boldsymbol{\Sigma}, \boldsymbol{\Theta}, \rho)=\ln \left|\mathbf{I}+\frac{\rho}{\rho \sigma_{p}^{2}+\sigma_{r}^{2}} \hat{\mathbf{H} \Sigma} \stackrel{\hat{\mathbf{H}}}{H}^{H}\right|
$$

2.2. Problem Formulation. We aim to maximize the harvested power by jointly designing the transmit signal, the $\mathrm{RC}$, and the PS ratio. To be specific, the problem is formulated as follows:

$$
\begin{gathered}
\max _{\Sigma \pm 0, \Theta, \rho} E(\Sigma, \Theta, \rho), \\
\text { s.t. } R(\Sigma, \Theta, \rho) \geq \eta, \\
\operatorname{Tr}(\boldsymbol{\Sigma}) \leq P_{s}, \\
\left|\Theta_{m, m}\right|=1,
\end{gathered}
$$




$$
0 \leq \rho \leq 1,
$$

where $\eta$ is the information rate threshold and $P_{s}$ is the maximum power for $\mathrm{Tx}$.

For any given $\{\Sigma, \Theta\}, \eta$ achieves the maximum when $\rho=0$. Thus, (5a) is feasible when the optimal value of the following problem is no less than $\eta$ :

$$
\begin{aligned}
& \max _{\Sigma \pm 0, \Theta} R(\boldsymbol{\Sigma}, \Theta, 0), \\
& \text { s.t. } \operatorname{Tr}(\boldsymbol{\Sigma}) \leq P_{s} .
\end{aligned}
$$

However, since $E(\Sigma, \Theta, \rho)$ and $R(\Sigma, \Theta, \rho)$ are nonconcave, (5) is nonconvex and hard to address. In the rest part, we assume that (5) is feasible and solve (5).

\section{An AO-Based Method for Problem (5)}

First, we define $\sigma^{2} \triangleq \sigma_{r}^{2}+\sigma_{p}^{2}$, $\omega^{2} \triangleq \sigma_{r}^{2} N_{T} / \sigma^{2}$, and $\widetilde{\mathbf{H}}=\hat{\mathbf{H}} / \sigma$. Since $\rho \sigma_{r}^{2}$ is commonly much smaller than $\sigma_{p}^{2}$, we omit this term in the rate expression and obtain the following problem:

$$
\begin{gathered}
\max _{\Sigma \pm 0, \Theta, \rho} \sigma^{2}(1-\rho)\left(\operatorname{Tr}\left(\widetilde{\mathbf{H} \Sigma} \widetilde{\mathbf{H}}^{H}\right)+\omega^{2}\right), \\
\text { s.t. } \ln \left|I+\rho \widetilde{\mathbf{H} \Sigma} \widetilde{\mathbf{H}}^{H}\right| \geq \eta, \\
(5 c)-(5 e) .
\end{gathered}
$$

It should be noted that, with fixed $\{\Sigma, \Theta\},(7)$ is convex with respect to (w.r.t.) $\rho$. In addition, with fixed $\{\rho, \Theta\}$, (7) is convex w.r.t. $\Sigma$. Both the two subproblems can be solved by the toolbox CVX [24].

The main difficulty in (7a) is the optimization of $\Theta$, since the unit modulus constraint (UMC) for $\Theta$ is nonconvex. Firstly, according to [15], solving (7) is equivalent to solving $\max _{\left|\Theta_{m, m}\right|=1} \operatorname{Tr}\left(\widetilde{\mathbf{H} \Sigma} \widetilde{\mathbf{H}}^{H}\right)$, which can be further expanded as

$$
\begin{aligned}
\operatorname{Tr}\left((\mathbf{H}+\mathbf{G} \Theta \mathbf{F}) \Sigma(\mathbf{H}+\mathbf{G} \Theta \mathbf{F})^{H}\right)= & \operatorname{Tr}\left(\mathbf{H} \Sigma \mathbf{H}^{H}\right)+2 \Re\left\{\operatorname{Tr}\left(\mathbf{G} \Theta \mathbf{F} \Sigma \mathbf{H}^{H}\right)\right\} \\
& +\operatorname{Tr}\left(\mathbf{G} \Theta \mathbf{F} \Sigma \mathbf{F}^{H} \Theta^{H} \mathbf{G}^{H}\right) .
\end{aligned}
$$

To further transform (8), we introduce the following lemma.

Lemma 1 (see [3]). Let $\mathbf{C}_{1} \in \mathbb{C}^{m \times m}, \quad \mathbf{C}_{2} \in \mathbb{C}^{m \times m}$, $\mathbf{E} \in \mathbb{C}^{m \times m}=\operatorname{Diag}\left(e_{1}, \ldots, e_{m}\right)$, and $e=\operatorname{diag}(\mathbf{E})$; then, the following relationships hold:

$$
\begin{aligned}
\operatorname{Tr}\left(\mathbf{E}^{H} \mathbf{C}_{1} \mathbf{E} \mathbf{C}_{2}\right) & =\mathbf{e}^{H}\left(\mathbf{C}_{1} \odot \mathbf{C}_{2}^{T}\right) \mathbf{e}, \\
\operatorname{Tr}\left(\mathbf{E C}_{2}\right) & =\mathbf{1}^{T}\left(\mathbf{E} \odot \mathbf{C}_{2}^{T}\right) 1=\mathbf{e}^{T} \mathbf{c}_{2}, \\
\operatorname{Tr}\left(\mathbf{E}^{H} \mathbf{C}_{2}^{H}\right) & =\mathbf{c}_{2}^{H} \mathbf{e}^{*},
\end{aligned}
$$

where $\mathbf{c}_{2}=\operatorname{diag}\left(\mathbf{C}_{2}\right)$.

Via Lemma 1, we have $\operatorname{Tr}\left(\mathbf{G} \Theta \mathbf{F} \Sigma \mathbf{F}^{H} \Theta^{H} \mathbf{G}^{H}\right)=\theta^{H} \mathbf{A} \theta$, $2 \mathfrak{R}\left\{\operatorname{Tr}\left(\mathbf{G} \Theta \mathbf{F} \Sigma \mathbf{H}^{H}\right)\right\}=2 \mathfrak{R}\left\{\mathbf{b}^{H} \theta\right\}$, where $\theta=\left[\theta_{1}, \ldots, \theta_{M}\right]^{T}$, $\mathbf{A}=\left(\mathbf{G}^{H} \mathbf{G}\right) \odot\left(\mathbf{F} \Sigma \mathbf{F}^{H}\right)^{T}$, and $\mathbf{b}=\operatorname{diag}\left(\mathbf{F} \Sigma \mathbf{H}^{H} \mathbf{G}\right)$, respectively.
Combining these equations, $\max _{\left|\Theta_{m, m}\right|=1} \operatorname{Tr}\left(\widetilde{\mathbf{H} \Sigma} \widetilde{\mathbf{H}}^{H}\right)$ is
equivalent to

$$
\max _{\left|[\theta]_{m}\right|=1, \forall m .} \boldsymbol{\theta}^{H} \mathbf{A} \theta+2 \mathfrak{R}\left\{\boldsymbol{\theta}^{H} \mathbf{b}\right\} .
$$

Then, by denoting $\mathbf{x}=\left[\begin{array}{ll}\theta^{H} & 1\end{array}\right]^{H}$, (10) can be equivalently rewritten as $\max _{\left|x_{m}\right|=1} f(\mathbf{x}) \triangleq \mathbf{x}^{H} \widetilde{A \mathbf{x}}$, where $\widetilde{\mathbf{A}}=\left[\begin{array}{cc}\mathbf{A} & \mathbf{b} \\ \mathbf{b}^{H} & 0\end{array}\right]$. Here, we develop the penalty-SDR method to solve the phase shifter design. To be specific, we introduce slack variable $\mathbf{X}=\mathbf{x} \mathbf{x}^{H}$, which means that $\mathbf{X} \pm 0$ and $\operatorname{rank}(\mathbf{X})=1$. Thus, we attain

$$
\begin{gathered}
\max _{X \pm 0} f(\mathbf{X}) \triangleq \operatorname{Tr}(\mathbf{X} \tilde{A}), \\
\text { s.t. } \ln |\mathbf{I}+\rho \mathbf{X} \widetilde{A}| \geq \eta, \\
\operatorname{diag}(\mathbf{X})=1, \\
\operatorname{rank}(\mathbf{X})=1 .
\end{gathered}
$$

Then, we handle the nonconvex (11c), where the following lemma is utilized.

Lemma 2 (see [25]). For any $\mathbf{B} \pm 0$, we have $|\mathbf{I}+\mathbf{B}| \geq 1+$ $\operatorname{Tr}(\mathbf{B})$ and the equality holds if and only if $\operatorname{rank}(\mathbf{B}) \leq 1$.

By Lemma 2, we have

$$
\operatorname{rank}(\mathbf{X})=1 \Leftrightarrow|\mathbf{I}+\mathbf{X}| \leq 1+\operatorname{Tr}(\mathbf{X}) \Leftrightarrow \ln |\mathbf{I}+\mathbf{X}| \leq \ln (2+M),
$$

where $\operatorname{Tr}(\mathbf{X})=M+1$.

However, $\ln |\mathbf{I}+\mathbf{X}|$ is concave w.r.t. $\mathbf{X}$. To handle this term, a penalty method is used by adding this term into (11). Then, (11) can be rewritten as

$$
\begin{gathered}
\max _{X \pm 0} \operatorname{Tr}(\mathbf{X} \tilde{A})-\kappa[\ln (2+M)-\ln (\mathbf{I}+\mathbf{X})], \\
\text { s.t. }(11 b),(11 c) .
\end{gathered}
$$

where $\kappa$ is a penalty factor to penalize any violation of $\operatorname{rank}(\mathbf{X})=1$. Moreover, $\ln |\mathbf{I}+\mathbf{X}|$ is concave w.r.t. $\mathbf{X}$ and thus can be upper bounded as

$$
\ln |\mathbf{I}+\mathbf{X}| \leq \operatorname{Tr}\left(\left(\mathbf{I}+\mathbf{X}^{n}\right)^{-1}\left(\mathbf{X}-\mathbf{X}^{n}\right)\right)+\ln \left|\mathbf{I}+\mathbf{X}^{n}\right|
$$

Based on (13), (14) can be written as

$$
\begin{gathered}
\max _{X \pm 0} \operatorname{Tr}(\mathbf{X} \tilde{A})-\kappa\left(\operatorname{Tr}\left(\left(\mathbf{I}+\mathbf{X}^{n}\right)^{-1}\left(\mathbf{X}-\mathbf{X}^{n}\right)\right)\right), \\
\text { s.t. }(11 b),(11 c) .
\end{gathered}
$$

(15) is convex w.r.t. $\mathbf{X}$ and hence it can be efficiently solved by CVX. A rank-one $\mathbf{X}^{*}$ can be obtained by solving (15) for a sufficiently small $\kappa$, and $\mathbf{x}^{*}$ can be attained by the eigendecomposition of $\mathbf{X}^{*}$. Besides, it is easily known that any rotation of $\mathbf{x}^{*}$ is also the optimal solution of (11). Thus, we rotate $\mathbf{x}^{*}$ by an angle $-\alpha$, where $\alpha$ is the phase of the last 


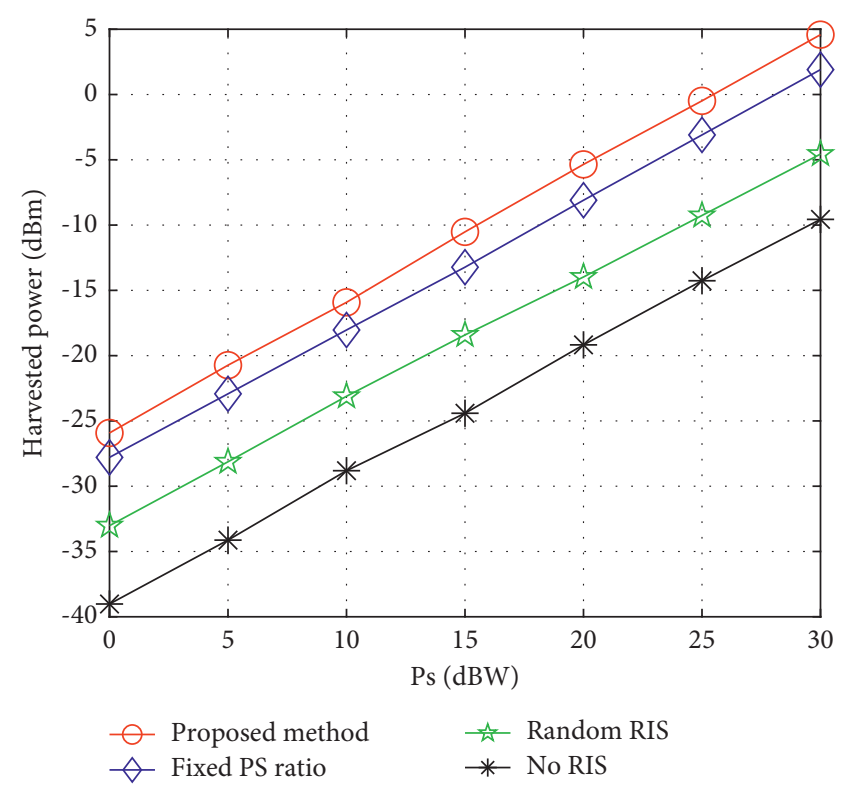

Figure 2: Harvested power versus $P_{s}$.

element of $\mathbf{x}^{*}$. Via this operation, we can obtain the optimal $\mathbf{x}^{*}$, where the last element is set to 1 and the rest elements constitute the vector $\theta^{*}$.

\section{Simulation Results}

Unless specified, the simulation setting are given as $P_{s}=10 \mathrm{dBW}, \quad N_{T}=N_{R}=5, \quad M=50, \quad \sigma_{r}^{2}=-80 \mathrm{dBm}$, $\sigma_{p}^{2}=-60 \mathrm{dBm}$, and $\eta=2 b p s / \mathrm{Hz}$. To active the EH circuit, we set the minimum $\mathrm{EH}$ threshold as $-40 \mathrm{dBm}$ [23]. The path loss is denoted as $L=L_{0}\left(d / d_{0}\right)^{-\beta}$, with $L_{0}=10^{-3}, d_{0}=1$, and $\beta$ as the path loss exponents. We set $\beta=-3$ for all links. In addition, we assume that the Tx-Rx link follows the Rayleigh fading, while the cascaded link follows the Rician fading with Rician factors 2.5 and 4, respectively. The coordinate of $\mathrm{Tx}, \mathrm{RIS}$, and $\mathrm{Rx}$ are set as $(0,5),(50,5)$, and $(50,0)$, respectively. Here, we compare the design with the following methods: (1) fixed PS ratio method, e.g., set the fixed $\rho=0.5$; (2) the random RIS method, e.g., choose the reflecting coefficient randomly; (3) the no RIS scheme. These methods are labeled as "Proposed method," "Fixed PS ratio," "Random RIS," and "No RIS," respectively.

First, Figure 2 plots the harvested power versus $P_{s}$, where we can see that the harvested power increases with $P_{s}$ for all these schemes. The proposed method achieves the best performance among these methods, while no RIS-assisted method suffers the worst performance. In addition, the fixed PS ratio design achieves better performance than random RIS design.

Then, Figure 3 shows the harvested power versus $M$, where we can see that with the increment of $M$, the harvested power increases for all these methods. However, our proposed design always obtains more harvested power than the other schemes, since with larger $M$, more signals can arrived at the RIS and the signals arrived at the $\mathrm{Rx}$ will increase, provided that the phase shifts are appropriately optimized.

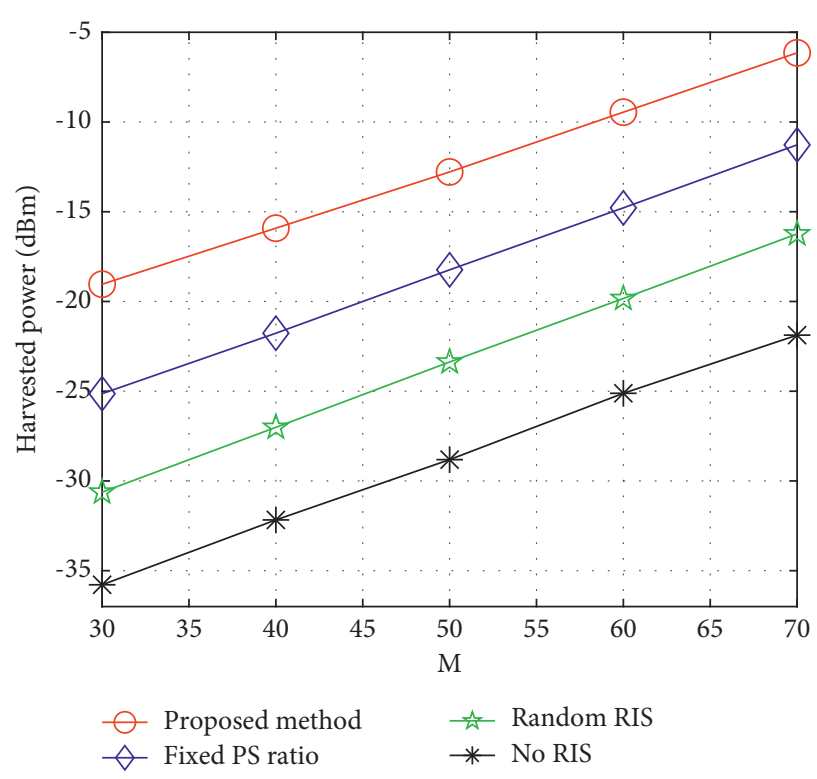

Figure 3: Harvested power versus $M$.

\section{Conclusion}

This work handled the joint design in a RIS-assisted MIMO system with SWIPT, where an AO algorithm has been proposed to maximize the harvested power and the phase shifter is solved by the penalty-based SDR method. Simulation results validated the ability of RIS in improving the wireless $\mathrm{EH}$ ability.

\section{Data Availability}

Data are available on request to the corresponding author.

\section{Conflicts of Interest}

The authors declare that there are no conflicts of interest regarding the publication of this article.

\section{Acknowledgments}

This work was supported by the Industry-University-Research Cooperation Project of Jiangsu Province under Grant no. BY2018282.

\section{References}

[1] Q. Wu, S. Zhang, B. Zheng, C. You, and R. Zhang, "Intelligent reflecting surface-aided wireless communications: a tutorial," IEEE Transactions on Communications, vol. 69, no. 5, pp. 3313-3351, 2021.

[2] G. Zhou, C. Pan, H. Ren, K. Wang, and A. Nallanathan, "Intelligent reflecting surface aided multigroup multicast MISO communication systems," IEEE Transactions on Signal Processing, vol. 68, pp. 3236-3251, 2020.

[3] S. Zhang and R. Zhang, "Capacity characterization for intelligent reflecting surface aided MIMO communication," IEEE Journal on Selected Areas in Communications, vol. 38, no. 8, pp. 1823-1838, 2020. 
[4] H. Niu, Z. Chu, F. Zhou, Z. Zhu, M. Zhang, and K. K. Wong, "Weighted sum secrecy rate maximization using intelligent reflecting surface," IEEE Transactions on Communications, vol. 69, no. 9, pp. 6170-6184, 2021.

[5] T. Shu, J. He, and L. Li, "Near-field passive localization and gain-phase compensation with partly calibrated arrays," IEEE Transactions on Aerospace and Electronic Systems, 2021.

[6] T. Shu, J. He, and V. Dakulagi, "3-D near-field source localization using a spatially spread acoustic vector sensor," IEEE Transactions on Aerospace and Electronic Systems, 2021.

[7] R. Wang, Z. Xing, and E. Liu, "Joint location and communication study for intelligent reflecting surface aided wireless communication system," 2021, https://arxiv.org/pdf/2103. 01063v1.pdf.

[8] S. Zeng, H. Zhang, B. Di, Z. Han, and L. Song, "Reconfigurable intelligent surface (RIS) assisted wireless coverage extension: RIS orientation and location optimization," IEEE Communications Letters, vol. 25, no. 1, pp. 269-273, 2021.

[9] F. Wen, J. Shi, and Z. Zhang, "Closed-form estimation algorithm for EMVS-MIMO radar with arbitrary sensor geometry," Signal Processing, vol. 186, Article ID 108117, 2021.

[10] G. Zheng, Y. Song, and C. Chen, "Height measurement with meter wave polarimetric MIMO radar: signal model and MUSIC-like algorithm," Signal Processing, vol. 190, Article ID 108344, 2021.

[11] F. Wang, H. Li, and J. Fang, "Joint Active and Passive Beamforming for IRS-Assisted Radar,” 2020.

[12] S. Buzzi, E. Grossi, M. Lops, and L. Venturino, "Foundations of MIMO Radar Detection Aided by Reconfigurable Intelligent Surfaces," 2021.

[13] X. Chen, J. C. Ke, W. Tang et al., "Design and implementation of MIMO transmission based on dual-polarized reconfigurable intelligent surface," IEEE Wireless Communications Letters, vol. 10, no. 10, pp. 2155-2159, 2021.

[14] E. Bjornson and L. Sanguinetti, "Power scaling laws and nearfield behaviors of massive MIMO and intelligent reflecting surfaces," IEEE Open Journal of the Communications Society, vol. 1, pp. 1306-1324, 2020.

[15] Z. Chen, Q. Shi, Q. Wu, and W. Xu, "Joint transceiver optimization of MIMO SWIPT systems for harvested power maximization," IEEE Signal Processing Letters, vol. 24, no. 10, pp. 1557-1561, 2017.

[16] S. Gong, Z. Yang, C. Xing, J. An, and L. Hanzo, "Beamforming optimization for intelligent reflecting surface-aided SWIPT IoT networks relying on discrete phase shifts," IEEE Internet of Things Journal, vol. 8, no. 10, pp. 8585-8602, 2021.

[17] J. Tang, D. K. C. So, N. Zhao, A. Shojaeifard, and K.-K. Wong, "Energy efficiency optimization with SWIPT in MIMO broadcast channels for internet of things," IEEE Internet of Things Journal, vol. 5, no. 4, pp. 2605-2619, 2018.

[18] K. Xu, Z. Shen, Y. Wang, X. Xia, and D. Zhang, "Hybrid timeswitching and power splitting SWIPT for full-duplex massive MIMO systems: a beam-domain approach," IEEE Transactions on Vehicular Technology, vol. 67, no. 8, pp. 7257-7274, 2018.

[19] Z. Chu, Z. Zhu, F. Zhou, M. Zhang, and N. A. Dhahir, "Intelligent reflecting surface assisted wireless powered sensor networks for internet of things," IEEE Transactions on Communications, vol. 69, no. 7, pp. 4877-4889, 2021.

[20] Q. Wu and R. Zhang, "Joint active and passive beamforming optimization for intelligent reflecting surface assisted SWIPT under QoS constraints," IEEE Journal on Selected Areas in Communications, vol. 38, no. 8, pp. 1735-1748, 2020.

[21] C. Pan, H. Ren, K. Wang et al., "Intelligent reflecting surface aided MIMO broadcasting for simultaneous wireless information and power transfer," IEEE Journal on Selected Areas in Communications, vol. 38, no. 8, pp. 1719-1734, 2020.

[22] C. He, X. Xie, K. Yang, and Z. J. Wang, "A Joint Power Splitting, Active and Passive Beamforming Optimization Framework for IRS Assisted MIMO SWIPT System," 2021, https://arxiv.org/pdf/2105.14545.pdf.

[23] H. Niu and L. Ni, "Intelligent reflect surface aided secure transmission in MIMO channel with SWIPT," IEEE Access, vol. 8, Article ID 192132, 2020.

[24] M. Grant and S. Boyd, "CVX: matlab software for disciplined convex programming," 2012, https://cvxr.com/cvx.

[25] S. Hong, C. Pan, H. Ren, K. Wang, K. K. Chai, and A. Nallanathan, "Robust transmission design for intelligent reflecting surface-aided secure communication systems with imperfect cascaded CSI," IEEE Transactions on Wireless Communications, vol. 20, no. 4, pp. 2487-2501, 2021. 